\title{
Fano interference in the optical absorption of an individual gold-silver nanodimer
}

Anna Lombardi ${ }^{1}$, Marcin P. Grzelczak ${ }^{2}$, Etienne Pertreux ${ }^{1}$, Aurélien Crut ${ }^{1}$, Paolo Maioli ${ }^{1}$, Isabel Pastoriza-Santos ${ }^{2}$, Luis M. Liz-Marzán ${ }^{2,3,4}$, Fabrice Vallée ${ }^{1}$ and Natalia Del Fatti ${ }^{*}$

${ }^{1}$ FemtoNanoOptics group, Univ Lyon, Université Claude Bernard Lyon 1, CNRS, Institut Lumière Matière, F69622 Villeurbanne, France

${ }^{2}$ Departamento de Química Física, Universidade de Vigo, 36310 Vigo, Spain

${ }^{3}$ Bionanoplasmonics Laboratory, CIC biomaGUNE, 20009 Donostia-San Sebastián, Spain

${ }^{4}$ Ikerbasque, Basque Foundation for Science, 48013 Bilbao, Spain

*email: natalia.del-fatti@univ-lyon1.fr

This file contains:

- Supporting Note 1 - Dipolar modeling of Fano effect in heterodimers

- $\quad$ Supporting Figures S1-S3 


\section{Supporting Note 1 - Dipolar modeling of Fano effect in heterodimers}

We analytically analyze the effect of Fano interference on the optical response of a heterodimer composed by two model nanospheres with resonant and constant absorption spectra, respectively. This is performed by considering simplified expressions for polarizabilities of the interacting nanoparticles and using a dipolar quasi-static approach. In this approximated framework, the spatial variation of the incident electromagnetic wave within the heterodimer components is neglected, and the response of each nanosphere in the dimer to the electromagnetic field is modeled as that of an induced dipole at the center of the sphere. Calculations yield the polarization-dependent effective polarizabilities $\alpha_{1,2}^{\text {eff }}$ of the two nanospheres composing the dimer, as well as the total polarizability of the dimer $\alpha=\alpha_{1}^{e f f}+\alpha_{2}^{e f f}$. For light polarized along the dimer axis and sufficiently large separation between the dimer components, one obtains ${ }^{1}$ :

$$
\left\{\begin{array}{l}
\alpha_{1}^{\text {eff }}=\alpha_{1}^{0}+C \frac{\alpha_{1}^{0} \alpha_{2}^{0}}{2} \\
\alpha_{2}^{\text {eff }}=\alpha_{2}^{0}+C \frac{\alpha_{1}^{0} \alpha_{2}^{0}}{2} \\
\alpha=\alpha_{1}^{0}+\alpha_{2}^{0}+C \alpha_{1}^{0} \alpha_{2}^{0}
\end{array}\right.
$$

with $C=1 /\left(\pi l^{3}\right)$, where $l$ is the center to center interparticle distance, and $\alpha_{1,2}^{0}$ are the polarizabilities of isolated nanospheres.

More specifically, we consider the interaction of two nanospheres respectively described by a Drude-like polarizability (sphere 1, with amplitude $\alpha_{01}$, resonant frequency $\omega_{R}$ and damping rate $\gamma<<\omega_{R}$ ) and a constant, purely imaginary one (sphere 2): 


$$
\left\{\begin{array}{l}
\alpha_{1}^{0}(\omega)=\alpha_{01} \frac{\omega_{R}^{2}}{\omega_{R}^{2}-\omega^{2}-i \omega \gamma} \\
\alpha_{2}^{0}=i \alpha_{02}
\end{array}\right.
$$

Introducing the reduced frequency $\xi=\frac{\omega-\omega_{R}}{(\gamma / 2)}$, the asymmetry factor (Fano parameter) $q=\frac{C \alpha_{02}}{2}$ and $A=\alpha_{01} \omega_{R} / \gamma$, the absorption cross-section of the whole dimer $\sigma_{a b s} \propto \operatorname{Im}(\alpha)$ is proportional to:

$$
\sigma_{a b s}(\xi) \propto \alpha_{02}+A\left(\frac{1-2 q \xi}{\xi^{2}+1}\right) \approx\left(\alpha_{02}+A\right)-A \frac{(\xi+q)^{2}}{\xi^{2}+1}
$$

the last expression being valid in the weak coupling assumption $(q<<1)$. It writes as the difference between a constant absorption and the $A \frac{(\xi+q)^{2}}{\xi^{2}+1}$ term, which corresponds to the pure Fano lineshape $\mathrm{e}^{2,3}$ with an asymmetry characterized by $q$ (a Lorentzian profile being retrieved in the $q \rightarrow 0$ limit, corresponding to $l \rightarrow \infty$, i.e., isolated dimer components).

The absorption cross-sections of the individual components within the dimer are then given by:

$$
\left\{\begin{array}{l}
\sigma_{a b s, 1}(\xi) \propto A\left(\frac{1}{\xi^{2}+1}-\frac{q \xi}{\xi^{2}+1}\right) \\
\sigma_{a b s, 2}(\xi) \propto \alpha_{02}-A \frac{q \xi}{\xi^{2}+1}
\end{array}\right.
$$

They are plotted, as well as the total one, in Figure S1. This figure shows that dipolar electromagnetic interaction results in (i) a spectral red shift of the total dimer absorption, as compared to non-interacting particles (solid vs dashed red lines), (ii) a weak modification of the resonant absorption spectrum of particle 1 when interacting with particle 2 , resulting in a 
quasi-Lorentzian shifted spectrum (solid vs dashed gray lines), (iii) the appearance of a large wavelength-dependence in the absorption of particle 2 , which evolves from an almost undispersed profile to a Fano-like one upon interaction with particle 1 (solid vs dashed orange lines). In the case of experimental conditions described in the main text, the evidenced Fanolike profile can be reproduced using Eq. 3 with $q \approx 0.6$ (Figure S1b).

All previous results can be transposed to the case of light polarized orthogonally to the dimer axis, which corresponds to a coupling constant $C$ (or $q$ ) of opposite sign and with a 2fold smaller amplitude.

\section{References}

(1) Lombardi, A.; Grzelczak, M. P.; Crut, A.; Maioli, P.; Pastoriza-Santos, I.; Liz-Marzán, L. M.; Del Fatti, N.; Vallée, F. ACS Nano 2013, 7, 2522-2531.

(2) Fano, U. Phys. Rev. 1961, 124, 1866-1878.

(3) Miroshnichenko, A. E.; Flach, S.; Kivshar, Y. S. Rev. Mod. Phys. 2010, 82, 2257. 

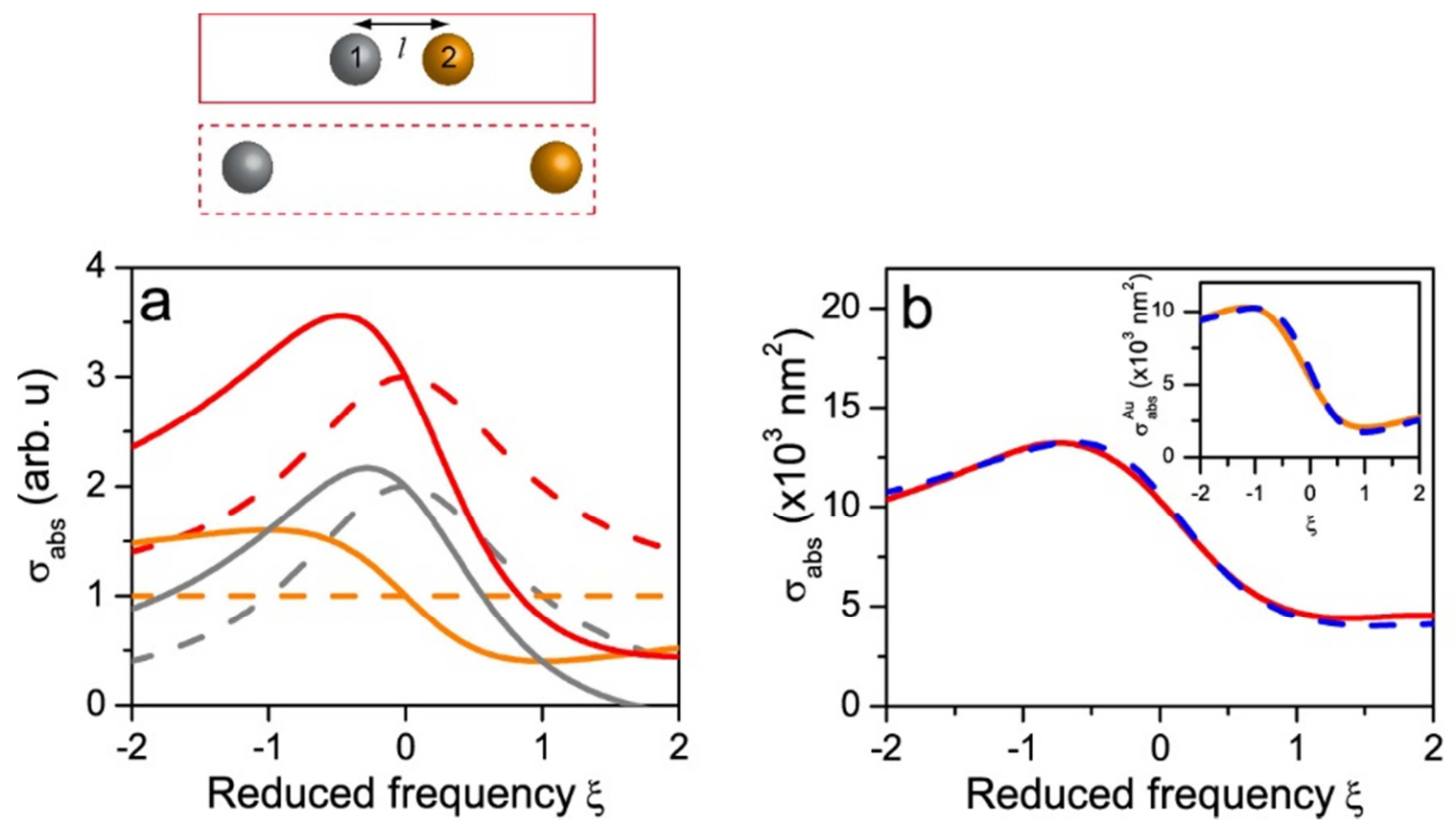

Figure S1. Simplified dipolar model for Fano interference effect in the absorption of a heterodimer. (a) Individual $\sigma_{a b s, 1}$ (gray), $\sigma_{a b s, 2}$ (orange) and total $\sigma_{a b s}=\sigma_{a b s, 1}+\sigma_{a b s, 2}$ (red) absorption cross-sections for isolated (dashed lines) and interacting (solid lines) nanospheres, using $A / \alpha_{02}=2$ and $q=0.6$. (b) Finite-element computed absorption of the dimer of Figure 1 (solid red line, as in Figure 1c of the main text) and its fit using Fano profile (corresponding to eq. 3 with $q \approx 0.6$, dashed blue line). Inset: the finite-element computed gold absorption (solid orange line, as in Figure 1c of the main text) is also reproduced by a Fano profile (eq. 4). Reduced frequencies were defined using $\omega_{R}$ and $\gamma$ values deduced from a Lorentzian fit of the absorption cross-section of the silver nanoparticle in the heterodimer. The $A$ parameter related to Ag SPR peak absorption was independently adjusted in the two fits to compensate for discrepancies between finite-element and dipolar calculations due to strong simplifications made in the latter. 


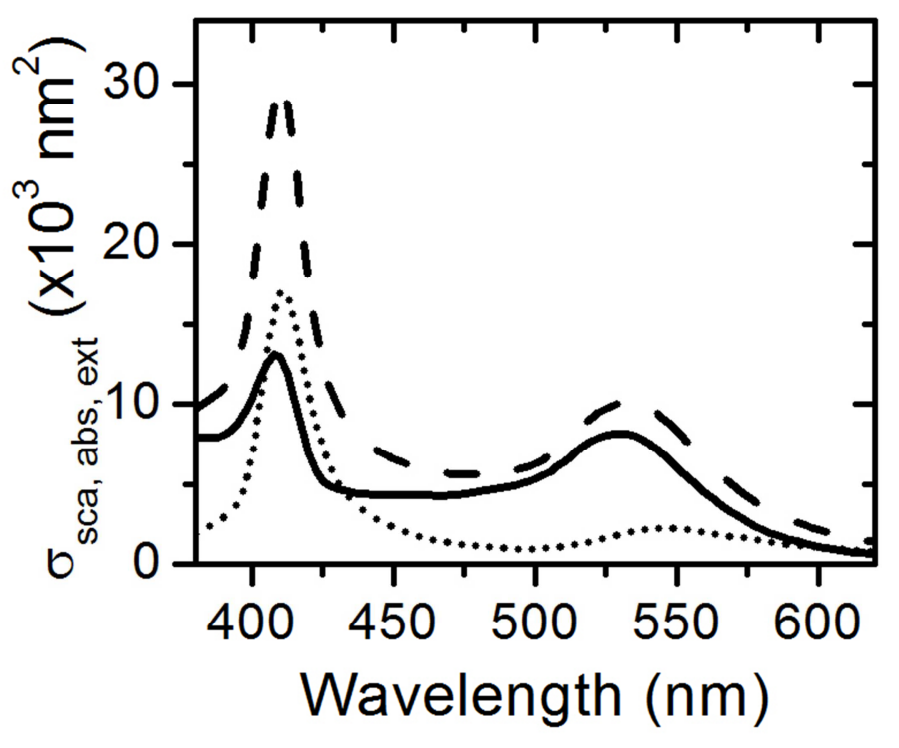

Figure S2. Linear optical spectroscopy of a single $\mathrm{Ag} @ \mathrm{SiO}_{2}-\mathrm{Au}$ nanodimer with light orthogonal to its axis. Computed cross-section spectra using TEM-determined morphology of the dimer of Figure 1 for incident light polarized orthogonal to its axis: extinction (dashed black), absorption (solid black) and scattering (dotted black). 

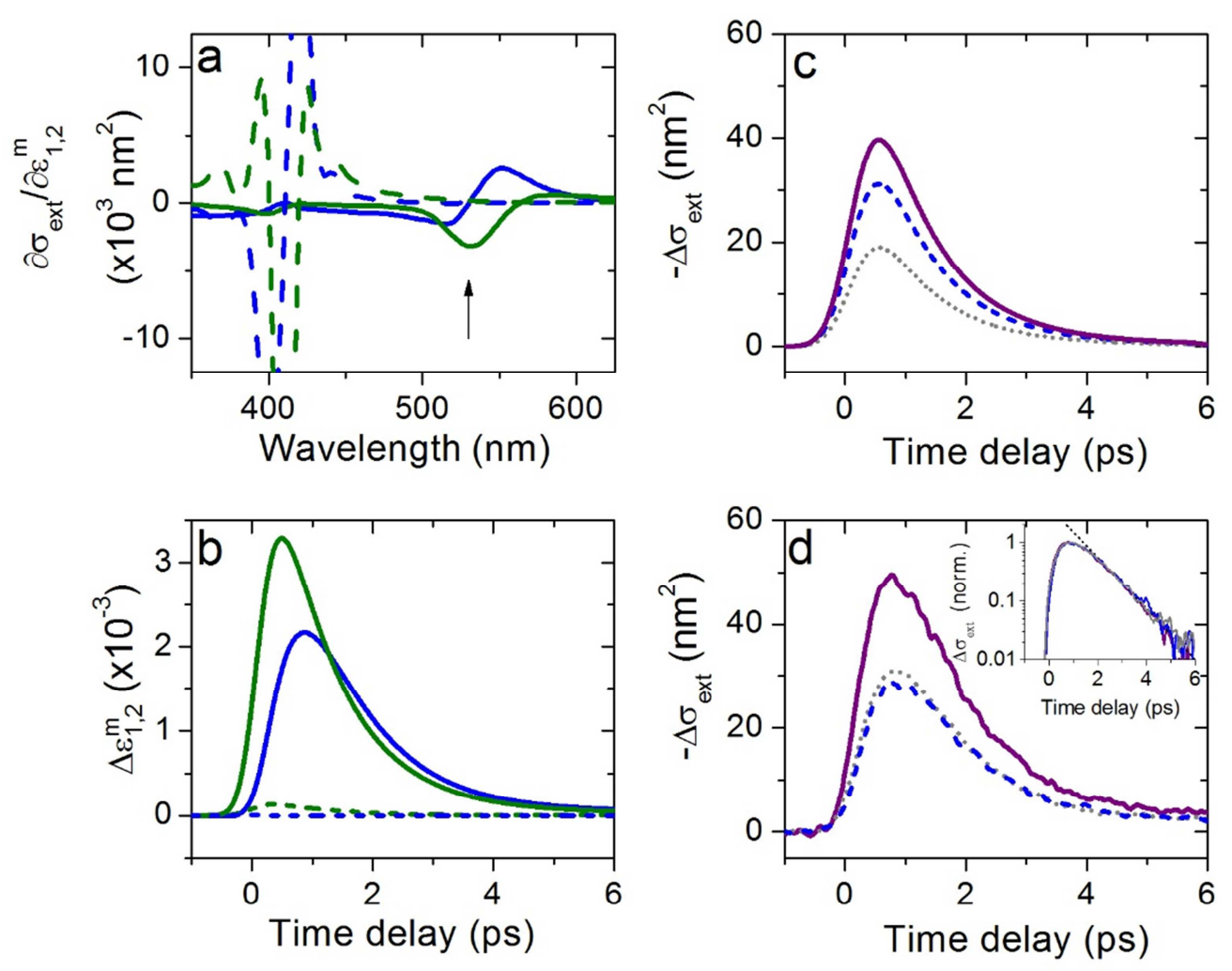

Figure S3. Modeling the ultrafast optical response of a single $\mathrm{Ag} @ \mathrm{SiO}_{2}-\mathrm{Au}$ nanodimer with light orthogonal to its axis. (a) Partial derivatives $\partial \sigma_{e x t} / \partial \varepsilon_{i}^{m}$ of the extinction crosssection (Eq. 2) of the dimer of Figure 1 numerically computed for light polarization orthogonal to its axis, for $i=1$ (blue line) and 2 (green lines), and $m=\mathrm{Au}$ (solid line) and $\mathrm{Ag}$ (dashed lines) cases. The arrow shows the experimental probe wavelength, $\lambda_{\text {pr }}=530 \mathrm{~nm}$. (b) Computed time evolution of the real (solid blue line) and imaginary (solid green line) part of the interband gold dielectric function change $\Delta \varepsilon_{1,2}^{A u}$ at $\lambda_{\mathrm{pr}}=530 \mathrm{~nm}$ for an electron temperature increase $\Delta T_{e x c}^{A u}=\Delta T_{e x c}^{r e f}=10 \mathrm{~K}$. Dashed lines: same for $\Delta \varepsilon_{1,2}^{A g}$. (c) Timedependent extinction changes of the dimer of Figure $1,-\Delta \sigma_{\mathrm{ext}}$, computed at $\lambda_{\mathrm{pr}}=530 \mathrm{~nm}$ for orthogonal polarization (eq. 2 with excitations corresponding to incident pump fluence $F_{\mathrm{pp}}=$ $1.2 \mu \mathrm{J} / \mathrm{cm}^{2}$ and orthogonal absorption cross-sections from Figure 1d), at different pump wavelengths around Fano resonance: $\lambda_{\mathrm{pp}}-\lambda_{\mathrm{SPR}}^{\mathrm{Ag}} \approx-25 \mathrm{~nm}$ (solid violet), $-15 \mathrm{~nm}$ (dashed blue) and $-5 \mathrm{~nm}$ (dotted gray). (d) Time-resolved extinction changes of the dimer of Figure 1, $\Delta \sigma_{\text {ext }}$, with same excitation and probing conditions as (c), deduced from experimental $\Delta T r / T r$ (Figure 2c). To account for slightly different spectral positions of silver-like SPR in experimental and computed linear extinction spectra, comparisons are made with respect to $\lambda_{\mathrm{SPR}}^{\mathrm{Ag}}=425 \mathrm{~nm}$ (experiments) and $420 \mathrm{~nm}$ (theory). Inset: normalized extinction changes on a logarithmic scale after subtraction of the long delay background. The relaxation time of the exponential decay (dotted line) is $\tau_{e-p h}=1 \mathrm{ps}$. 